\title{
Magnitude estimation of softness
}

\author{
Robert M. Friedman · Kim D. Hester · Barry G. Green • \\ Robert H. LaMotte
}

Received: 22 April 2008 / Accepted: 16 July 2008 / Published online: 5 August 2008

(C) The Author(s) 2008

\begin{abstract}
The human capacity to estimate the magnitude of softness of silicone rubber disks of differing compliance was studied under experimental conditions that altered the mode of contact. Subjects were able to scale softness regardless of whether they (1) actively indented each specimen by tapping or pressing it with the finger pad, (2) received passive indentation of the finger pad by each specimen via a force controlled tactile stimulator, thus eliminating kinesthetic cues, or (3) actively indented each specimen with a stylus that was manipulated either by tapping with one finger, or held by two fingers in a precision grip, thereby removing tactile cues provided by direct mechanical contact between the finger pad and specimen. Ratings of softness were independent of moderate variations in peak compressional force and force-rate. Additionally, functions
\end{abstract}

\author{
R. M. Friedman · K. D. Hester · R. H. LaMotte $(\bowtie)$ \\ Department of Anesthesiology, \\ Yale University School of Medicine, P.O. Box 208051, \\ 333 Cedar Street, New Haven, CT 06520, USA \\ e-mail: robert.lamotte@yale.edu \\ R. M. Friedman \\ e-mail: robert.friedman@vanderbilt.edu \\ Present Address: \\ R. M. Friedman \\ Department of Psychology, Vanderbilt University, \\ Nashville, TN, USA \\ B. G. Green \\ John B. Pierce Laboratory, New Haven, CT, USA \\ B. G. Green \\ Department of Surgery (Otolaryngology), \\ Yale School of Medicine, New Haven, CT, USA
}

for scaling softness were affected by the mode of contact; the slopes of the functions were greater in the tasks with a complete complement of compliance cues. When subjects were asked to classify objects as either hard or soft, specimens were classified as soft if the compliance were greater than that of the human finger. This suggests that the classification of softness depends on whether the object conforms to the body, and that tactile information about the spatial profile of object deformation is sufficient for the magnitude scaling of softness. But typically, kinesthetic information about the magnitude of object displacement, along with contact vibratory cues is also used while judging softness especially in the absence of direct skin contact with the object when using a tool.

Keywords Compliance - Finger pad - Tool use · Active touch · Passive touch · Psychophysics

\section{Introduction}

Softness is a fundamental dimension of the tactual perception of objects. Softness is the perceptual correlate of compliance, which is defined as the amount of deformation caused by an applied force. In the simplest sense, the perception of softness would therefore require knowledge of the deformation of an object and the associated contact forces. However, studies of the ability to discriminate compliance have shown that information related to these variables can be obtained in a variety of ways. For example, the discrimination of differences in the compliance of objects can occur despite differences in the types of sensory cues available (LaMotte 2000; Srinivasan and LaMotte 1995). When actively touching objects with a finger pad, subjects can easily discriminate small differences in compliance 
despite moderate variations in displacements and contact forces (Srinivasan and LaMotte 1995). In tasks where a compliant object is applied to a passive finger pad, subjects discriminate differences in compliance in the absence of kinesthetic cues even when peak force and velocity are randomly varied to eliminate cues of compressional force-rate. Findings like these suggest the importance of spatial tactile cues from the skin in perceiving the softness of objects.

However, such cues are not necessarily required because subjects discriminate just as well by contacting compliant objects with a tool as with a finger pad (LaMotte 2000). Thus, the somatosensory system derives cues about force and displacement, components of compliance, from a number of sources including efferent commands, sensory signals obtained from muscles, joints and skin and prior knowledge of the sensory cues evoked when objects of differing compliance contact the skin.

The capacity of humans to scale the magnitude of softness has received little attention. The role of kinesthetic and tactile cues in scaling softness is unknown as are the effects of different modes of contact. These factors are not investigated in the single earlier study on the scaling of the hardness and softness of objects (Harper and Stevens 1964). For example, it is not known how the perceived magnitude of softness is affected by differences in the peak compressional force or rate of force of indentation between the skin and the object or whether contact is brought about actively or passively or by means of a tool. Another question that has not been addressed is what criteria are used to categorize an object as hard or soft.

The present study investigated the capacities of humans to scale the magnitude of softness of objects of differing compliance applied to the finger pad. The objects not only included those that were stiffer than the finger pad, as used in prior studies of softness (Srinivasan and LaMotte 1995; LaMotte 2000), but also those that were more compliant. To determine which sensory cues are used to evaluate the magnitude of softness of an object, we investigated the effects of eliminating or separately varying tactile and kinesthetic sensory cues delivered to the finger pad. Subjects either actively indented each specimen with the finger pad or received passive indentation of the skin to eliminate kinesthetic cues. Alternatively, specimens were actively indented by means of a stylus to eliminate cutaneous contact cues.

\section{Materials and methods}

\section{Subjects}

Twenty-two subjects, ranging in age from 18 to 41 years, gave informed consent to the psychophysical protocol approved by the Yale Institutional Review Board.
Specimen preparation

Compliant specimens were made from two-component silicone rubber compounds (RTV615, RTV6126, RTV6136, and RTV6156, General Electric, Waterford, NY) to achieve specimens with a wide range of compliances. Except for RTV615, the two component compounds were mixed in varying proportions of the silicone rubber compound (part A) and curing agent (part B). For the RTV615 compound, parts $A$ and $B$ were mixed in a ratio of 10:1 and various amounts of a diluent ( $47 \mathrm{~V}$ Silicone Fluid, The Smith Group, Inc., Warminster, PA) were added to produce specimens differing in compliance. After mixing, the solutions were poured into Petri dishes $(35 \mathrm{~mm}$ diameter by $10 \mathrm{~mm}$ deep) at a slow rate to prevent the formation of air bubbles. The silicone solution solidified after 12-24 h of curing in an incubator set at $50^{\circ} \mathrm{C}$. Contact surfaces were powdered with talc to eliminate surface cues (e.g. stickiness) associated with the silicone specimens.

\section{Measurement of compliance}

Compliance was determined by measuring the amount of force produced by indenting each specimen with a cylindrical probe as a function of probe displacement (Srinivasan and LaMotte 1995). In brief, each specimen was placed on a rigid platform attached to a load cell (Honeywell Sensotec, Columbus, $\mathrm{OH})$. A flat-end cylindrical probe $(10 \mathrm{~mm}$ diameter) mounted to a stepper motor was applied to the center of a specimen at a constant velocity of $0.5 \mathrm{~mm} / \mathrm{s}$ until a displacement of about $7 \mathrm{~mm}$ or a force of $1.25 \mathrm{~N}$ was reached. The compliance of the finger pad was measured with the same device. Displacement was measured with a linear variable differential transformer sensor, (Schaevitz Sensors, Hampton, VA). The compliance of a specimen (and finger) was taken as the ratio of the amount of its deformation to an applied force of $1 \mathrm{~N}$. The compliances of the eight specimens used in the present study spanned a range, in progressive log units of 1.3 , from 0.21 to $7.59 \mathrm{~mm} / \mathrm{N}$ (Fig. 1). The objects in the present study included objects that were more compliant than the finger pad as well as those that were stiffer. In contrast, objects previously used to study softness discrimination (LaMotte 2000; Srinivasan and LaMotte 1995) were all stiffer than the finger pad.

\section{Experimental paradigms}

Subjects judged the magnitude of softness of each specimen in a series of five tasks that differed in the type of sensory information available to a subject (Fig. 2). During each task, a drape prevented a subject from viewing his or her hand and specimen. 


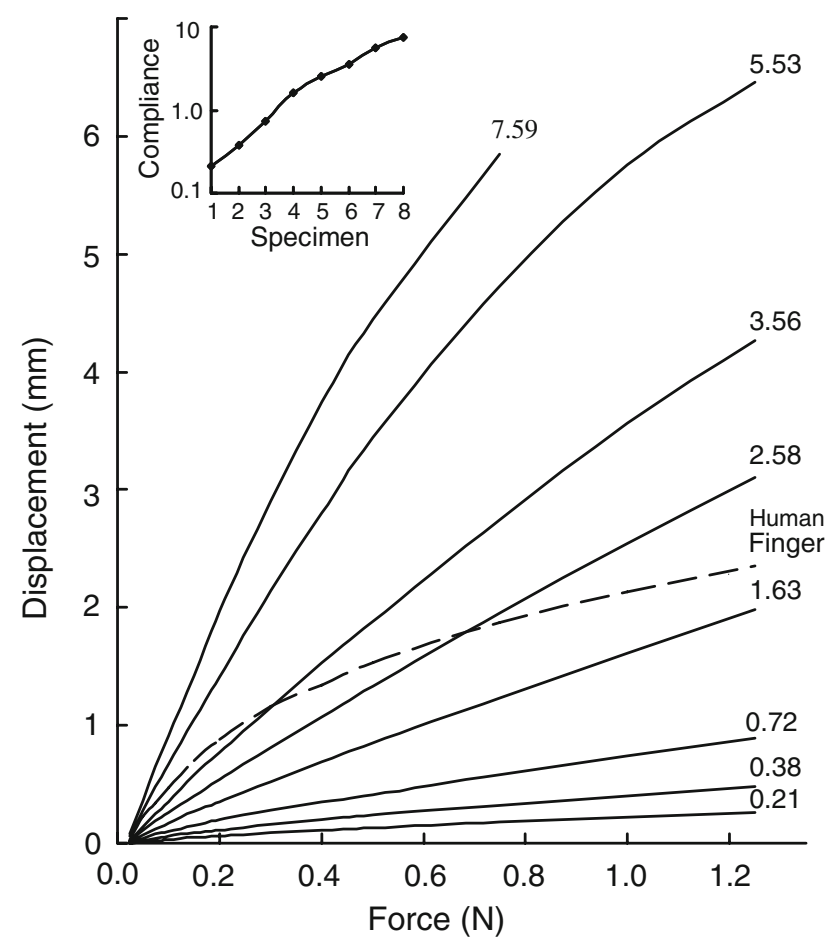

Fig. 1 Measurements of the compliance of the rubber specimens and the finger pad. Each specimen and the finger pad of a human subject were indented with a flat-ended cylindrical probe of $10 \mathrm{~mm}$ diameter at a constant velocity of $0.5 \mathrm{~mm} / \mathrm{s}$ as both force and displacement were recorded. Compliance (in $\mathrm{mm} / \mathrm{N}$ ), defined as the slope of the function relating displacement to force, is provided to the right of the curve for each specimen (solid lines) or the middle finger pad (dashed line). Inset: Each specimen is separated from the adjacent one by $1.3 \mathrm{log}$ units and ranked from the hardest (1) to the softest (8)

Subjects were instructed to judge the magnitude of softness of each specimen by moving a computer mouse that caused a cursor to move along a visual analog scale presented on a video screen. The scale consisted of a vertical line the bottom of which was labeled as "No Softness" and the top as "Softest Imaginable." After bringing the cursor to a desired level, a subject pressed the left mouse button to select or lock a position. If a subject wanted to change a selection after the first left mouse button press, a press of the right mouse button released the cursor and allowed a subject to select a different position along the scale. After selecting a position along the scale, a second press of the left mouse button confirmed a selection and ended a trial.

\section{Stimulus presentation}

The specimens were presented one at a time, at an interstimulus interval of $30 \mathrm{~s}$, in pseudorandom order such that each was presented three times in a session of 24 trials. At the onset of the trial, signaled by a warning tone, the subject actively indented (or passively received) the specimen and then rated its magnitude of softness.
Active indentation with a finger

In the active indentation task, in which both kinesthetic and cutaneous tactile cues were available, the subject's hand rested on a slotted platform $3 \mathrm{~cm}$ above a specimen that was mounted on a load cell (Fig. 2A). The subject indented the center of a specimen with the left middle finger, starting from a position just above the specimen, using a natural but moderate amount of force, and then retracting the finger with one smooth, continuous motion. If during practice trials a subject indented a specimen with too much force $(>5 \mathrm{~N})$, the subject was trained to apply less.

In one experiment, subjects were instructed to press the specimen with the middle finger (termed "Active Pressing with a Finger"). In another, the subjects were told to tap the specimen ("Active Tapping with a Finger"). From subjective observations made by the subjects, it appeared that the tactile cues were of two types: those derived from the contact mechanics of the initial impact between skin and specimen ("impact tactile cues"), and cues subsequently provided by a changing pressure distribution on the skin as the finger indented the deformable object ("pressure distribution cues").

Active tapping with a tool

A tool (12 mm diameter) grasped between the thumb and forefinger (a "two finger stylus") was used to tap the specimen (Fig. 2B). The stylus was capped with a hemisphere, $8 \mathrm{~mm}$ in diameter that was used to contact the specimen. In other experiments, a tool designed to be manipulated with a single finger (a "one-finger stylus"), was mounted perpendicular to an arm that pivoted on the shaft of a torque motor (Fig. 2C). Each end of the stylus was capped with a hemisphere, $8 \mathrm{~mm}$ in diameter. The hemisphere at one end rested on the distal pad of the subject's middle finger. The hemisphere at the other end contacted the specimen. A load cell recorded the normal forces exerted by the stylus on the specimen. In each of these tapping tasks, kinesthetic cues were available but tactile cues from the skin were restricted to signals derived from the impact between the tool and the specimen rather than the specimen itself.

Passive pressing of the finger

The back of a subject's finger was positioned at an angle of $30^{\circ}$ and fixed to a holder with double-sided tape (Fig. 2D). The specimen was mounted to one end of a lever. The other end of the lever was attached to a servo-controlled torque motor (Aurora Scientific, Aurora, Canada). The torque motor, under program control (LabVIEW, National Instruments Corp., Austin, TX), was used to control the compressional force of the specimen against the finger pad. The 

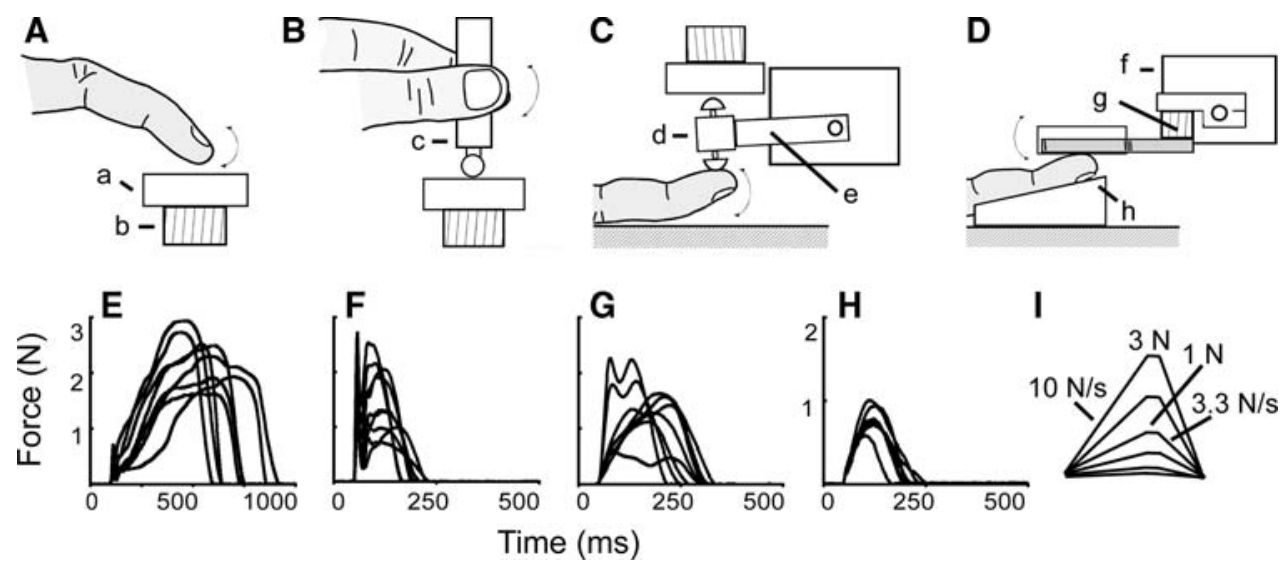

Fig. 2 Subjects scaled softness in five tasks. A Active tapping or pressing with the finger pad. Each specimen $(a)$ was mounted to a load cell $(b)$ that was used to measure compressional force. $B$ Active tapping with a two-finger tool. The unconstrained, two-finger stylus $(c)$ was tapped once against each specimen. The stylus had a shaft diameter of $12 \mathrm{~mm}$ and a sphere, $8 \mathrm{~mm}$ in diameter, at the end that contacted the specimen. $C$ Active tapping with an one-finger tool: The one-finger stylus $(d)$ had tip diameters of $8 \mathrm{~mm}$ at each end. The stylus was attached to a lever $(e)$ that pivoted on the shaft of a torque motor. The motor was used to exert a constant downward force of $10 \mathrm{mN}$ on the finger pad. This served to maintain contact between the tip of the stylus and the finger pad. $D$ Passive pressing of the finger. A torque motor $(f)$

amplitudes of the plateaus and the ramp rates of the trapezoidal waveforms used to press the specimens onto the finger pad were chosen from the rates and amplitudes of force traces subjects produced while actively indenting the specimens (Fig. 2E, F).

Subjects were asked to scale softness under conditions where peak force and force-rate were co-varied. The range of compressional forces was $0.15-3.0 \mathrm{~N}$ and force-rates ranged from 1 to $10 \mathrm{~N} / \mathrm{s}$ (Fig. 2I). Prior to the delivery of a force waveform, the specimen was slowly brought down to obtain a very light basal contact with the skin. This basal level of contact was shown to be insufficient for the identification or discrimination of specimens (data not shown). After this brief contact of 3-4 s duration, the motor pressed the specimen against the finger pad and then removed it from the skin. Each of a series of stimuli was applied with varied rates and peak forces of indentation. The range of forces delivered was restricted for the specimens of greater or lesser compliance (Table 1), as the greater forces required to indent the hardest objects would be excessive for the softest (causing the finger to reach the back of the Petri dish) and the lowest forces that were required for the softest objects would not be sufficient to indent the hardest. Subjects were instructed to ignore differences in force and force-rate and judge only the softness of a specimen. Stimuli were presented one at a time in a random order with an interstimulus interval of $30 \mathrm{~s}$. Each session consisted of 24 trials. The eight different specimens were presented to a subject in three pseudorandom blocks of trials.

pressed a specimen against a subject's finger pad via a lever $(g)$ that was attached to the shaft of the motor. The dorsum of the middle finger was held against a holder $(h)$ with double-sided tape. E-I Compressional forces measured for the five tasks during contact with specimen that had a compliance of $2.58 \mathrm{~mm} / \mathrm{N}$. E Active pressing with the finger (A). $F$ Active tapping with the finger $(A) . G$ Active tapping with the two-finger stylus $(B)$. $H$ Active tapping with the one-finger-stylus $(C)$. $I$ Passive pressing of the finger pad $(D)$. The motor held the specimen against the finger pad with a downward force of $1 \mathrm{~g}(9.8 \mathrm{mN})$ prior to pressing it against the skin with one of 6 trapezoidal waveforms of compressional force

Table 1 Compressional forces used for each specimen pressed against the passive finger pad

\begin{tabular}{|c|c|c|c|c|c|c|}
\hline \multirow{2}{*}{$\begin{array}{l}\text { Specimen } \\
\text { compliance } \\
(\mathrm{mm} / \mathrm{N})\end{array}$} & \multicolumn{6}{|c|}{ Compressional force $(\mathrm{N})$} \\
\hline & 0.15 & 0.30 & 0.60 & 1.00 & 2.00 & 3.00 \\
\hline 0.21 & & & & $*$ & $*$ & $*$ \\
\hline 0.38 & & & $*$ & $*$ & $*$ & $*$ \\
\hline 0.72 & & & $*$ & $*$ & $*$ & $*$ \\
\hline 1.63 & & $*$ & $*$ & $*$ & $*$ & $*$ \\
\hline 2.53 & $*$ & $*$ & $*$ & $*$ & $*$ & $*$ \\
\hline 3.56 & $*$ & $*$ & $*$ & $*$ & * & \\
\hline 5.58 & $*$ & $*$ & $*$ & $*$ & & \\
\hline 7.59 & $*$ & $*$ & $*$ & & & \\
\hline
\end{tabular}

\section{Categorization of objects}

With his/her arm and hand hidden from view by a drape, the subject used the middle finger to press objects of differing size, shape, texture and compliance. Subjects were instructed to categorize an object as either hard or soft based on everyday experience. Within a collection of 14 novel objects, the 8 silicone specimens used in the softness rating tasks were randomly presented and also categorized as either hard or soft. The goal was to obtain categorizations of hardness or softness for the 8 silicone specimens. The purpose of the novel objects served solely 
to anchor the categorization of the specimens within a context consisting of other kinds of objects differing in compliance.

Data and statistical analysis

For each subject and for each specimen presented in a given task, a mean was obtained of the magnitude estimates, i.e., numerical values corresponding to positions chosen along the scale or vertical line. A psychophysical function was obtained by plotting the mean of the log mean magnitude estimates obtained for all the subjects for each specimen as a function of the compliance of each specimen.

The force trace generated during active indentation of a specimen by either a finger or tool was evaluated to determine possible force cues used by subjects to rate softness. Measured aspects of the force trace included its peak and its rate, the latter calculated by dividing the peak by the time taken to reach the peak.

The statistical significance of differences between means obtained under different experimental conditions was evaluated using a two-factor (specimen compliance $\times$ task) repeated measures ANOVA. Comparisons between levels of a factor were performed with a Tukey test. The probability criterion for significance was 0.05 .

\section{Results}

Active indentation with the finger pad

Each subject's magnitude estimates of softness increased monotonically as a function of compliance regardless of whether the specimens were pressed or tapped with the finger pad (Fig. 3a, b). The log-linear slopes of the functions were 0.462 and 0.428 for pressing and tapping, respectively. For a given mode of contact (pressing or tapping) the means of the geometric mean estimates obtained for each subject (Fig. 3c) differed significantly for specimen compliance. However, there were no significant differences between the two modes of contact (two-way repeated measures ANOVA).

The contact force of the finger with each specimen, as measured by the load cell, differed for pressing and tapping. As illustrated for the force profiles generated by a subject while pressing or tapping a specimen that had a compliance of $2.58 \mathrm{~mm} / \mathrm{N}$, pressing (Fig. 2E) evoked longer latency to peak force than tapping (Fig. 2F) and a longer duration of contact. For each specimen, the mean peak compressional force and the mean rate of change in force (from contact to peak force) were calculated for each subject (Fig. 3d, e, g, h). The differences in means (across subjects) as a function of specimen compliance and task were evaluated using
Fig. 3 Perceived softness as a function of the compliance of each specimen actively tapped or pressed with the finger pad. a Magnitude estimates of softness obtained from individual subjects while pressing the specimens. b Magnitude estimates of softness obtained from individual subjects while tapping the specimens. c Mean magnitude estimates of softness obtained from tapping and pressing the specimens. d-i The peak compressional forces plotted for each subject as a function of compliance and obtained during pressing (d) and tapping (e) and averaged for all subjects (f). Similarly presented are the individual force rates for pressing (g) and tapping (h) and the means for all subjects (i)
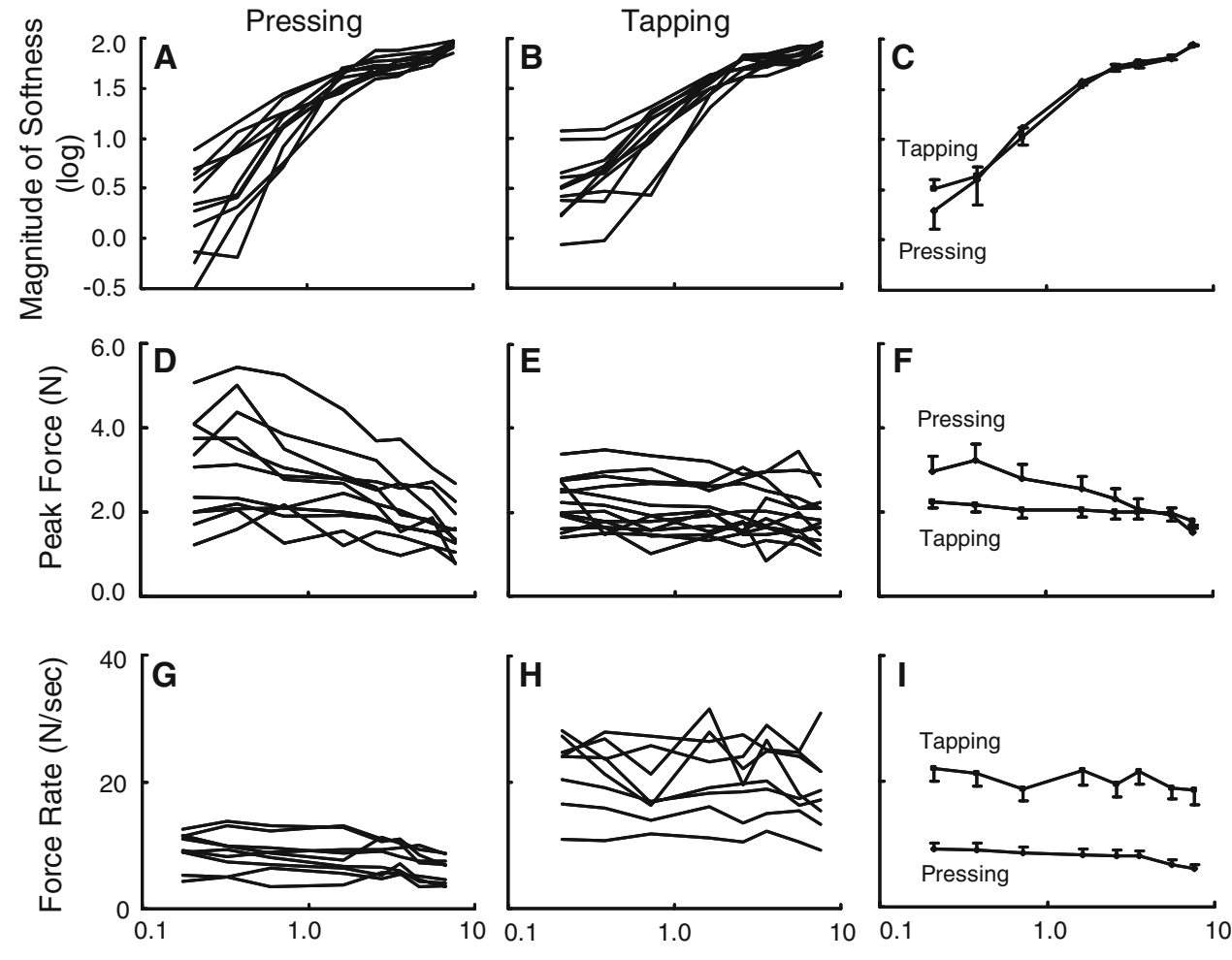

Compliance of Specimen $(\mathrm{mm} / \mathrm{N})$ 
two-way repeated measures ANOVAs. Subjects applied significantly greater force when pressing as opposed to tapping the three hardest specimens. Comparable forces were obtained when pressing or tapping the more compliant specimens (Fig. 3d-f). In contrast, for all specimens, the rate of change in force was significantly greater for tapping than for pressing (Fig. 3g-i). Despite these differences in the rate and magnitude of force applied, the magnitude scaling of softness was similar for the two tasks (Fig. 3c).

Subjects were observed to cluster into two groups, in that subjects tended to indent specimens with forces either greater or lower than $2.5 \mathrm{~N}$. Consequently we investigated the hypothesis that greater compressional forces, possibly generating more perceptible deformations of a specimen, would result in greater ratings of softness. We divided the psychophysical functions of subjects into two groups according to whether the subjects tended to press the more compliant specimens with peak compressional forces that were greater or lesser than $2.5 \mathrm{~N}$ (Fig. $3 \mathrm{~d}$ ). Subjects that used higher peak compressional forces to press the specimens rated the less compliant specimens as softer (Fig. 4); however this distinction did not hold for the softer specimens, where the softness ratings were comparable to the two subgroups of subjects (ANOVA, Force $\times$ Specimen; Tukey tests yielding significant pair wise differences only for specimens 0.38 and $0.72 \mathrm{~mm} / \mathrm{N}$ ). Thus, the mode of contact could affect softness ratings. To confirm that the differences in the softness functions were not peculiar to the individual subjects, we found no differences in softness

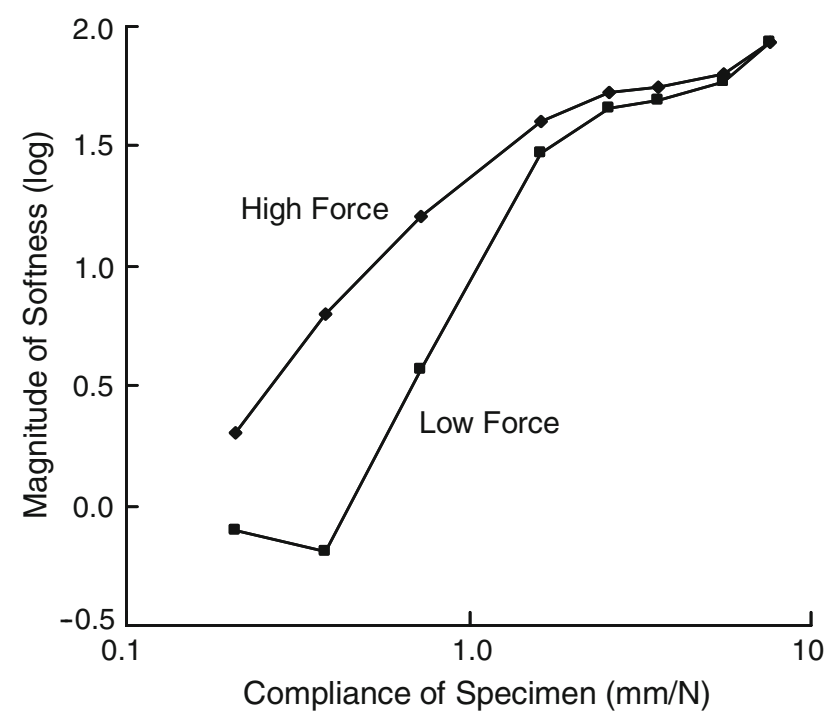

Fig. 4 Effects of compressional force on the perceived softness of specimens actively pressed with the finger pad. The magnitude estimation functions from subjects were divided into two natural groups, based on whether a subject tended to indent specimens with forces either greater or lower than 2.5 N. (Fig. 3d)
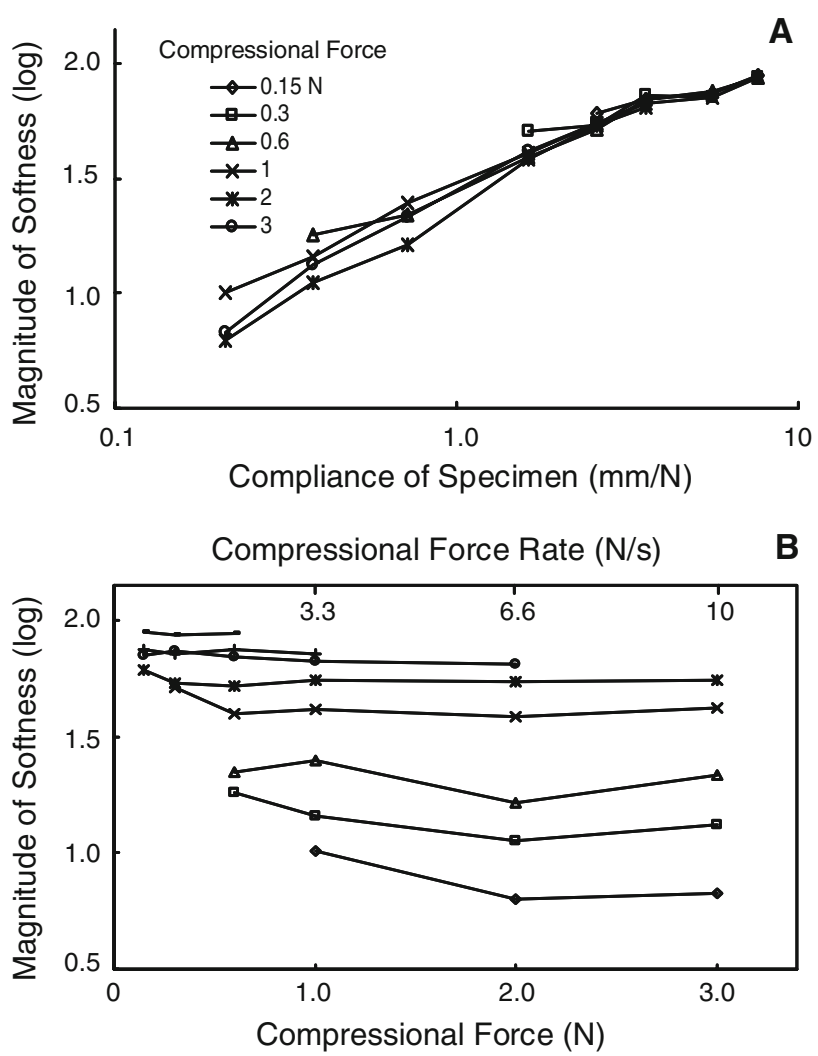

Fig. 5 Perceived softness as a function of the compliance of each specimen pressed onto the passive finger pad. a Mean magnitude estimates of softness for indentations of the specimens for each of the six compressional forces. b Relationship between magnitude estimates of softness and peak compressional force (or force-rate) for each specimen

ratings for the same division of subjects for the tapping task (ANOVA).

Indentation of the passive finger pad

In this task, because the specimens were pressed against the restrained, passive finger pad and the initial contact between specimen and skin was made slowly and under low force, there were neither kinesthetic cues nor cutaneous "impact" cues. Under these conditions, softness ratings increased linearly with compliance. Significantly different mean ratings of softness were obtained for different specimens (Fig. 5a, ANOVA). Specimens of greater compliance were rated as significantly softer than those of lesser compliance, except for the three least compliant specimens and the specimens with compliances of 3.56 versus $5.58 \mathrm{~mm} / \mathrm{N}$ (all other Tukey post-hoc ps $<0.05$ ). The scaling of softness was independent of differences in peak compressional force or force rate (Fig. 5b, RMANOVA). Thus, subjects were able to rate the magnitude of softness independent of kinesthetic, impact, or cues of peak compressional force or force-rate. 
Fig. 6 Perceived softness as a function of the compliance of each specimen actively indented with a stylus. a Magnitude estimates of softness of individual subjects who were required to use one finger to tap each specimen with a stylus b Magnitude estimates of softness of individual subjects who were required to tap each specimen with a stylus held in a precision grip. c Mean magnitude estimates of softness for the two modes of tapping. d-f Peak compressional forces as a function of compliance obtained from each subject during tasks that required tapping either with the one-finger stylus (d) or the two-finger stylus (e) and averaged for all subjects for each of these tasks (f)
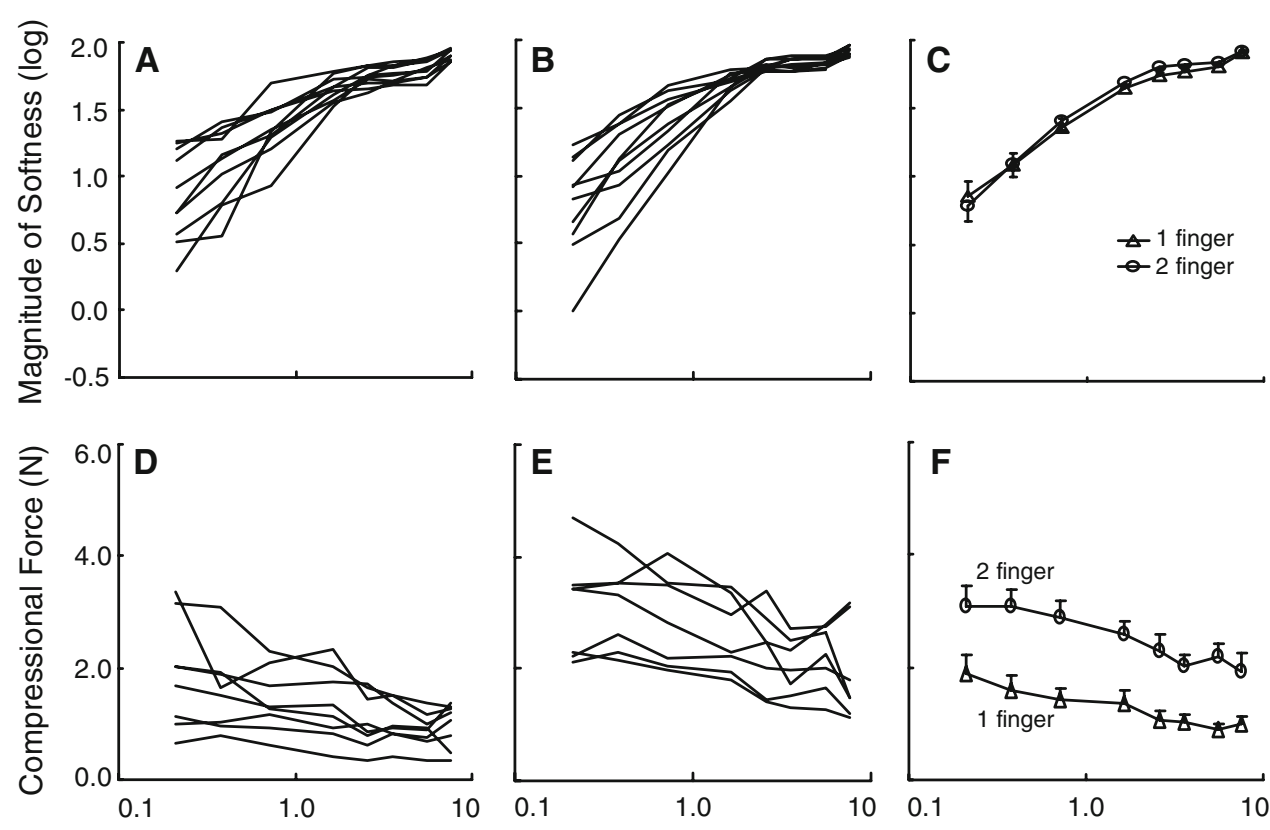

Compliance of Specimen $(\mathrm{mm} / \mathrm{N})$
Active tapping with a tool

When subjects actively indented the specimens using a stylus, softness ratings increased linearly with compliance in both the one- and two-finger tasks with respective slopes of 0.289 and 0.308 (Fig. 6a, b). There were no significant differences in the mean ratings of softness for the two tasks (Fig. 6c, ANOVA). The peak compressional forces subjects applied to specimens in each task increased with decreasing compliance and were consistently greater for the two-finger than the one-finger task (Figs. 2G, H, 6d-f, RMANOVA). Yet neither the differences in applied force delivered in each task, the differences in movement required, nor the presence or absence of shear or compressional forces of the tool against the finger pad(s) affected the ability of subjects to scale softness.

\section{Comparisons of softness ratings for the different tasks}

The mean magnitude ratings of softness were compared for all five tasks (Fig. 7) using a two-way ANOVA (task, specimen) and Tukey pairwise comparisons. In comparison with the other three tasks, actively tapping or pressing the specimens with a finger pad led to lower softness judgments but only for the harder specimens (ANOVA indicating significant task $\times$ specimen interactions). The slope of the function representing the mean ratings obtained for actively tapping and actively pressing the specimens was notably steeper $(0.445)$ than the slope of the function obtained for passive indentation (0.296) or the slope of the

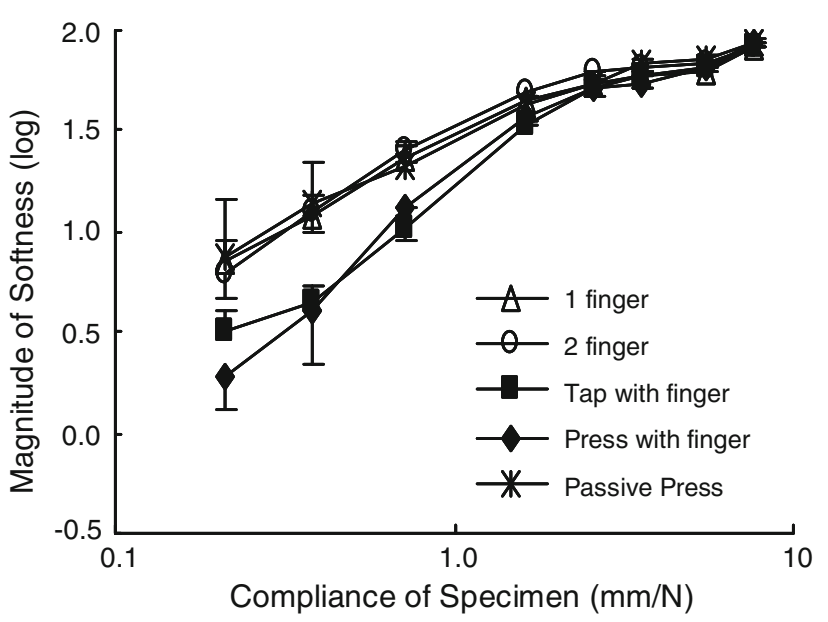

Fig. 7 Mean magnitude estimates of softness for the five different modes of contact. Individual estimates from each subject were averaged for each specimen during tasks that differed according to whether the specimen was pressed against the restrained finger pad ("Passive press") or actively tapped or pressed with the finger pad ("Tap with finger", "Press with finger") or actively tapped with a stylus controlled by one- or two fingers ("1 finger", "2 finger")

function representing the mean ratings obtained for active indentations with a stylus manipulated with one versus two fingers (0.298). Thus, the optimal psychophysical function for judgments of the magnitude of softness was obtained when there is direct contact between an actively moving finger and the specimen thereby allowing a full complement of sensory cues related to compliance. 


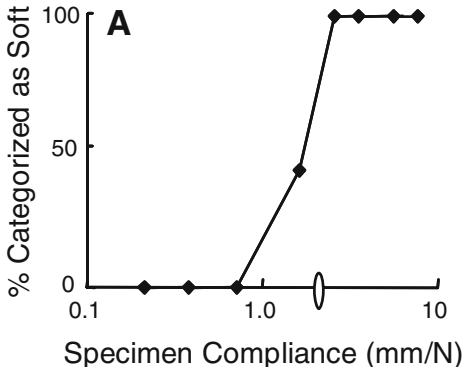

Specimen Compliance $(\mathrm{mm} / \mathrm{N})$

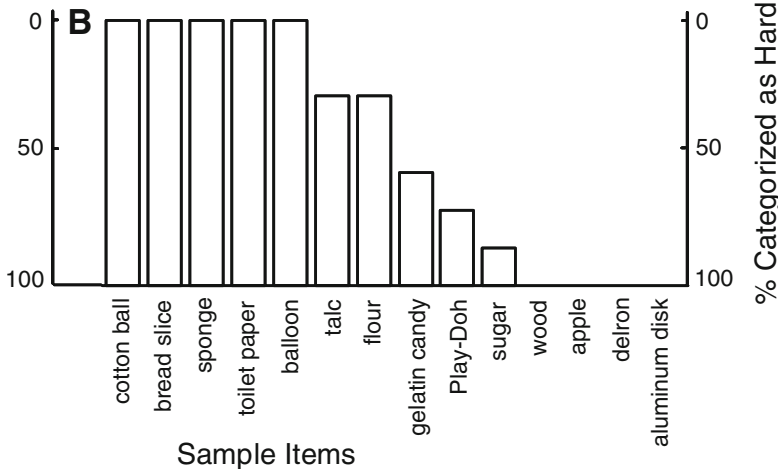

Sample Items

"talc", "flour") were poured into empty Petri dishes and loosely covered with plastic wrap. Seven subjects were instructed to press each object once with the middle finger pad. The specimens and household objects were intermixed and pseudorandomly presented. Specimens with compliances of $0.72 \mathrm{~mm} / \mathrm{N}$ or less were categorized as hard; specimens with compliances of $2.58 \mathrm{~mm} / \mathrm{N}$ or more were categorized as soft. The vertical ellipse marks the compliance of the finger pad

criminate the compliance of objects with direct contact of a finger pad, actively or passively, (Srinivasan and LaMotte 1995) or though indirect contact by means of a tool (LaMotte 2000).

When subjects categorized the specimens and common, household objects as either "hard" or "soft", specimens with compliances of $0.72 \mathrm{~mm} / \mathrm{N}$ or less were always categorized as hard (Fig. 8a, b). Specimens with compliances of $2.58 \mathrm{~mm} / \mathrm{N}$ or greater were always categorized as soft. The specimen with a compliance of $1.63 \mathrm{~mm} / \mathrm{N}$ was rated as soft by $43 \%$ of the subjects. For comparison purposes, the compliance of the human finger was measured as $2.14 \mathrm{~mm} / \mathrm{N}$. Because the breakpoint in categorizing an object as hard or soft corresponded to the compliance of the finger pad, the classification may be based on whether the object is more or less compliant than the finger pad.

\section{Discussion}

Subjects were able to rate softness under tasks that differed in the mode of contact and thus provided different sensory cues of compliance. Whether through active indentation with a finger or stylus, or passive pressing of a finger, softness ratings increased with specimen compliance. Softness ratings were comparable despite moderate variations in the peak compressional force and force-rate and/or the availability of sensory cues that differed both within and across tasks. However, the slopes of the softness scaling functions were steeper in the tasks that offered a complete complement of sensory cues. These results, obtained for the scaling of softness, extend those previously obtained for the discrimination of softness, where subjects were able to dis-
Cues in tasks used for the scaling of softness

Softness ratings for the active finger-tapping and pressing tasks were different from those for the passive or stylus tasks. The specimens of lower compliance were judged to be less soft (harder) in the task requiring active indentation with the finger pad than they were in the other tasks. Consequently, the slopes of the softness functions were steeper when actively indenting with a finger than when passively or actively indenting the specimen with a tool. Thus, softness judgments were influenced by the mode of contact, a variable known to influence the haptic identification of objects (Lederman and Klatzky 2004).

When actively contacting compliant objects with the finger pad, a full complement of compliance cues were available, and subjects made comparable judgments of softness whether tapping or pressing. Kinesthetic cues from muscle, joint, skin displacement were available to provide information about the displacement of both the finger and the specimen. In addition, tactile impact cues were generated by the initial impact between the skin and the specimen. Furthermore, tactile cues were provided by the changing pressure distribution ("pressure distribution cues") on the skin as the finger contacted and deformed the specimen. Peak compressional force and force-rate did not appear to be significant cues, because softness ratings were similar in the presence of large variations in peak compressional forces and force-rates, indicating that softness ratings 
were made independently of the force profiles of indentation. However, the less compliant specimens were rated as softer when indented with greater force. In this case, the use of greater compressional forces to deform an object provided additional "pressure distribution cues" for rating softness.

These cues could have been proved by mechanoreceptors on the side of the finger that have been shown to respond to objects indenting the center of a finger pad (Birznieks et al. 2001; Bisley et al. 2000; Khalsa et al. 1998). It is possible that higher softness ratings of the less compliant specimens at higher forces of indentation could be a consequence of the recruitment of laterally positioned mechanoreceptors. If a mean rate code is being used to code softness, the additional contribution of these mechanoreceptors might dilute the population response at high force levels leading to higher softness ratings. Perhaps anesthetizing the sides of the finger pad and then having subjects rate softness of specimens with varying forces of indentation might help to assess the contribution of laterally located mechanoreceptors.

When the specimen was pressed onto the passive finger pad, the only sensory cues available were those of a changing pressure distribution on the skin. Kinesthetic cues were absent as were vibrotactile signals produced by the impact of an object against the skin. Under this condition, the perceived softness of specimens was not influenced by variations in the peak compressional force and the rate of change in force. This suggests that subjects evaluated softness by attending only to the spatial distribution of pressure on the finger pad, without interference from the force profile.

When a tool was used to contact the specimen, kinesthetic and contact cues were available but tactile cues were restricted only to those obtained from the impact of the tool against the specimen. Because similar softness ratings were obtained when a stylus was either tapped with one finger or held between two fingers in a precision grip, we can eliminate shear or compressional forces of the tool against the finger pad(s) as necessary cues to scale softness with a tool. Similarly, since the peak compressional forces used in the two tasks were different, the absolute compressional force could not be the sole cue for softness. Instead, for compliance to be sensed via a tool, it is likely that tactile force cues must be supplemented by vibratory signals produced upon impact of the tool with the object and by kinesthetic cues related to the velocity and depth of indentation.

Thus, combinations of kinesthetic and tactile cues typically contribute to the perception of softness; no single cue appears to be necessary. Another conclusion is that ratings of specimen softness are independent of peak compressional force or force-rate. That softness ratings are independent of the rate of indentation is supported by a published finding that judgments of the magnitude of compressional force produced by a stiff probe were not influenced by changes in the rate of indentation (Pare et al. 2002).

Cues used to judge softness

Although the present findings indicate that different cues can be used to judge the compliance of a surface in different tasks, a central question is what cues are most important under normal conditions of active touch. Direct contact with an object provides information about the shape of the object and the change in shape as pressure is applied. A likely clue for softness is the change in the pressure distribution or change in finger displacement over time, since softness judgments are independent of compressional force-rate. Thus, a natural cue for softness is the dynamic change in object shape as a function of time. In contrast, hardness is the resistance to changes in shape in the face of changes in contact force. In a related study, Peine and Howe (1998) found that finger displacement, not pressure distribution, correlated with detection of a hard ball buried in a compliant medium, suggesting that finger displacement rather than object displacement may be a cue for hardness.

Results from the categorization task and the profile of the softness rating function support the view that the compliance of the finger pad is the breakpoint for categorizing an object as soft or hard. That is, an object is classified as soft if it conforms to the body and hard if the body conforms to the object. This leads to the possibility that subjects use different kinds of sensory information to assess soft versus hard objects. Whereas softness might depend on the degree to which the object conforms to the body, hardness might be related to the degree to which the body conforms to the object.

Acknowledgments We would like to thank Michael Naiyeju, Kenneth W. Greenquist and Joseph T. Hester PhD for their contributions to the research.This work was supported by a grant from NINDS NS37609.

Open Access This article is distributed under the terms of the Creative Commons Attribution Noncommercial License which permits any noncommercial use, distribution, and reproduction in any medium, provided the original author(s) and source are credited.

\section{References}

Birznieks I, Jenmalm P, Goodwin AW, Johansson RS (2001) Encoding of direction of fingertip forces by human tactile afferents. J Neurosci 21:8222-8237

Bisley JW, Goodwin AW, Wheat HE (2000) Slowly adapting type I afferents from the sides and end of the finger respond to stimuli on the center of the fingerpad. J Neurophysiol 84:57-64

Harper R, Stevens SS (1964) Subjective hardness of compliant materials. Q J Exp Psychol 16:204-215

Katz D (1938) The judgements of test bakers. A psychological study. Occup Psychol 12:139-148 
Khalsa PS, Friedman RM, Srinivasan MA, Lamotte RH (1998) Encoding of shape and orientation of objects indented into the monkey fingerpad by populations of slowly and rapidly adapting mechanoreceptors. J Neurophysiol 79:3238-3251

LaMotte RH (2000) Softness discrimination with a tool. J Neurophysiol 83:1777-1786

Lederman SJ, Klatzky RL (2004) Haptic identification of common objects: effects of constraining the manual exploration process. Percept Psychophys 66:618-628
Pare M, Carnahan H, Smith AM (2002) Magnitude estimation of tangential force applied to the fingerpad. Exp Brain Res 142:342348

Peine WJ, Howe RD (1998) Do humans sense finger deformation or distributed pressure to detect lumps in soft tissue? In: Proceedings of ASME dynamic systems and control division, Anaheim, CA, vol DSC-64, pp 273-278

Srinivasan MA, LaMotte RH (1995) Tactual discrimination of softness. J Neurophysiol 73:88-101 